\title{
ON THE FRIEDRICHS OPERATOR
}

\author{
PENG LIN AND RICHARD ROCHBERG
}

(Communicated by Palle E. T. Jorgensen)

\begin{abstract}
Let $\Omega$ be a simply connected domain in $\mathbb{C}^{1}$ with the area measure $d A$. Let $\bar{P}_{\Omega}$ be the orthogonal projection from $L^{2}(\Omega, d A)$ onto the closed subspace of antiholomorphic functions in $L^{2}(\Omega, d A)$. The Friedrichs operator $\bar{T}_{\Omega}$ associated to $\Omega$ is the operator from the Bergman space $L_{a}^{2}(\Omega)$ into $L^{2}(\Omega, d A)$ defined by $\bar{T}_{\Omega} f=\bar{P}_{\Omega} f$. In this note, some smoothness conditions on the boundary of $\Omega$ are given such that the Friedrichs operator $\bar{T}_{\Omega}$ belongs to the Schatten classes $S_{p}$.
\end{abstract}

\section{INTRODUCTION}

Let $\Omega$ be a connected open set in the complex plane $\mathbb{C}^{1}$ and let $d A$ denote the area measure. Let $L^{2}(\Omega)=L^{2}(\Omega, d A)$ be the usual Lebesgue space, $L_{a}^{2}(\Omega)$ the Bergman space consisting of holomorphic functions in $L^{2}(\Omega)$ and $\overline{L_{a}^{2}(\Omega)}=$ $\left\{\bar{f}: f \in L_{a}^{2}(\Omega)\right\}$. Let $P_{\Omega}$ be the orthogonal projection from $L^{2}(\Omega)$ onto $L_{a}^{2}(\Omega)$ and $\bar{P}_{\Omega}$ the orthogonal projecton from $L^{2}(\Omega)$ onto $\overline{L_{a}^{2}(\Omega)}$. Let $K_{\Omega}(z, w)$ denote the Bergman kernel of $\Omega$. Then

$$
\begin{aligned}
P_{\Omega} f(z) & =\int_{\Omega} K_{\Omega}(z, w) f(w) d A(w), \\
\bar{P}_{\Omega} f(z) & =\int_{\Omega} \overline{K_{\Omega}(z, w)} f(w) d A(w) \\
& =\int_{\Omega} K_{\Omega}(w, z) f(w) d A(w) .
\end{aligned}
$$

According to [Sh2], the Friedrichs operator associated to $\Omega$ is defined by

$$
T_{\Omega} f=P_{\Omega} \bar{f} \quad \text { for } \quad f \in L_{a}^{2}(\Omega) .
$$

Note that $T_{\Omega}$ is bounded and real-linear, but not complex-linear since

$$
T_{\Omega}(\lambda f)=\bar{\lambda} T_{\Omega} f, \quad \lambda \in \mathbb{C} .
$$

Observe that $\overline{T_{\Omega} f}=\overline{P_{\Omega} \bar{f}}=\bar{P}_{\Omega} f$. We introduce the operator $\bar{T}_{\Omega}$ which is

Received by the editors January 24, 1994 and, in revised form, March 9, 1994 and April 4, 1994.

1991 Mathematics Subject Classification. Primary 47B10, 47B38; Secondary 32H10, 46E35.

The second author's work was supported in part by a grant from NSF.

(C)1995 American Mathematical Society 
defined by

$$
\bar{T}_{\Omega} f=\bar{T}_{\Omega} f=\bar{P}_{\Omega} f \quad \text { for } \quad f \in L_{a}^{2}(\Omega) .
$$

Then $\bar{T}_{\Omega}$ is a complex-linear operator. It is obvious that $T_{\Omega}^{2}$ is also a complexlinear operator.

If $\Omega=\mathbb{D}$, the unit disk, then it is easy to check that $T_{\mathbb{D}}$ (and $\bar{T}_{\mathbb{D}}$ too) is a rank-one operator. But for general $\Omega, T_{\Omega}$ (or $\bar{T}_{\Omega}$ ) may even not be compact. In 1937, Friedrichs proved the following compactness theorem.

Theorem (Friedrichs, see [Sh2]). If $\Omega$ is a bounded connected domain in $\mathbb{C}^{1}$ with $C^{1, \alpha}$ boundary, then $T_{\Omega}$ (or equivalently $\bar{T}_{\Omega}$ ) is compact.

In [Sh1], Shapiro gave a characterization of the finite-rank Friedrichs operator $T_{\Omega}$ (or equivalently $\bar{T}_{\Omega}$ ) by using the notion of quadrature domain. In [F] and [N], Friedrichs and Norman studied the essential spectrum of $T_{\Omega}^{2}$ in cases where $T_{\Omega}^{2}$ is not compact. As one can see from the above, the spectral properties of the Friedrichs operator are closely related to the geometry of $\Omega$.

The problem that we are interested in here is the following.

Problem. When are $\bar{T}_{\Omega}$ and $T_{\Omega}^{2}$ in the Schatten classes $S_{p}$ ?

In this note we will concentrate on simply connected domains in $\mathbb{C}^{1}$. To state our main results, we need some definitions and notation.

The Schatten class $S_{p}(1 \leq p<\infty)$ consists of all the compact operators $T$ from a Hilbert space $H_{1}$ to another Hilbert space $H_{2}$ for which the singular numbers $s_{n}(T)$ form a sequence belonging to $l^{p}$. The singular numbers of the operator $T$ are the eigenvalues of $\left(T^{*} T\right)^{1 / 2}$.

For $s \in \mathbb{Z}_{+}, \alpha>0$ and $1 \leq p<\infty$, the weighted Sobolev space $W_{p}^{s, \alpha}(\mathbb{D})$ on the unit disk is defined to be the completion of $C^{\infty}(\overline{\mathbb{D}})$ with respect to the norm

$$
\|f\|_{W_{p}^{s, \alpha}(\mathbb{D})}=\left(\sum_{\substack{\xi+\eta \leqslant s \\ \xi, \eta \in \mathbb{Z}_{+}}}\left\|\left(\frac{\partial}{\partial z}\right)^{\xi}\left(\frac{\partial}{\partial \bar{z}}\right)^{\eta} f\right\|_{L^{p}\left(\mathbb{D},\left(1-|z|^{2}\right)^{\alpha-1} d A(z)\right)}^{p}\right)^{1 / p} .
$$

Note that when $\alpha=1, W_{p}^{s, 1}(\mathbb{D})=W_{p}^{s}(\mathbb{D})$, which is the usual Sobolev space.

A Jordan curve $\Gamma$ is of class $C^{n, \alpha}(n=1,2, \cdots, 0<\alpha<1)$ if it has a parametrization $\Gamma: w(\tau): 0 \leq \tau \leq 2 \pi$ that is $n$ times continuously differentiable and satisfies $w^{\prime}(\tau) \neq 0$ and $\left|w^{(n)}\left(\tau_{1}\right)-w^{(n)}\left(\tau_{2}\right)\right| \leq C\left|\tau_{1}-\tau_{2}\right|^{\alpha}$.

Main Theorem. Let $\Omega$ be a proper, simply connected domain in $\mathbb{C}^{1}$ and let $\varphi$ be the conformal mapping of $\mathbb{D}$ onto $\Omega$. Then:

(1) For $1<p<\infty$, if $\varphi^{\prime} / \overline{\varphi^{\prime}} \in W_{p}^{1, p-1}(\mathbb{D})$, then $\bar{T}_{\Omega} \in S_{p}$.

(2) For $p=1$, if $\varphi^{\prime} / \overline{\varphi^{\prime}} \in W_{1}^{2, \gamma}(\mathbb{D})$ for some $0<\gamma<1$, then $\bar{T}_{\Omega} \in S_{1}$.

From the Main Theorem we have

Corollary A. If $\Omega$ is a bounded simply connected domain in $\mathbb{C}^{1}$ with $C^{2, \alpha}(0<$ $\alpha<1)$ boundary, then $\bar{T}_{\Omega}$ belongs to all $S_{p}$ for $1<p<\infty$.

Corollary B. If $\Omega$ is a bounded simply connected domain in $\mathbb{C}^{1}$ with $C^{3, \alpha}(0<$ $\alpha<1)$ boundary, then $\bar{T}_{\Omega} \in S_{1}$.

For $T_{\Omega}^{2}$ we have similar results. 


\section{CoMPACTNESS}

In this section we will give some other conditions for the compactness of the Friedrichs operator. For the bounded simply connected domain case, Friedrichs' compactness theorem [F] will be a corollary of our results.

As we will see in the following lemma, the Friedrichs operator is closely related to the small Hankel operator $h_{b}$ on the unit disk $\mathbb{D}$. Given $b \in L^{2}(\mathbb{D})$, the small Hankel operator $h_{b}$ (with symbol $b$ ) is defined by

$$
h_{b} f=\bar{P}_{\mathbb{D}}(b f) \quad \text { for } f \in L_{a}^{2}(\mathbb{D}) .
$$

Lemma 2.1. Let $\Omega$ be a proper, simply connected domain in $\mathbb{C}^{1}$ and let $\varphi$ be the conformal mapping of $\mathbb{D}$ onto $\Omega$. Then

$$
\bar{T}_{\Omega}=V_{\varphi^{-1}} h_{\overline{\varphi^{\prime}} / \varphi^{\prime}} U_{\varphi}=V_{\varphi^{-1}} h \frac{}{P_{\mathbf{D}}\left(\varphi^{\prime} / \overline{\varphi^{\prime}}\right)} U_{\varphi},
$$

where $U_{\varphi}: L_{a}^{2}(\Omega) \rightarrow L_{a}^{2}(\mathbb{D}), U_{\varphi} f=f \circ \varphi \cdot \varphi^{\prime}$ and $V_{\varphi^{-1}}: \overline{L_{a}^{2}(\mathbb{D})} \rightarrow \overline{L_{a}^{2}(\Omega)}$, $V_{\varphi^{-1}} g=g \circ \varphi^{-1} \cdot \overline{\left(\varphi^{-1}\right)^{\prime}}$.

Proof. From p. 33 of $[B]$ we have

$$
K_{\Omega}(w, z)=K_{\mathbb{D}}\left(\varphi^{-1}(w), \varphi^{-1}(z)\right)\left(\varphi^{-1}\right)^{\prime}(w) \overline{\left(\varphi^{-1}\right)^{\prime}(z)} .
$$

Then for any $f \in L_{a}^{2}(\Omega)$,

$$
\begin{aligned}
\bar{T}_{\Omega} f(z) & =\bar{P}_{\Omega} f(z)=\int_{\Omega} K_{\Omega}(w, z) f(w) d A(w) \\
& =\int_{\Omega} K_{\mathbb{D}}\left(\varphi^{-1}(w), \varphi^{-1}(z)\right)\left(\varphi^{-1}\right)^{\prime}(w) \overline{\left(\varphi^{-1}\right)^{\prime}(z)} f(w) d A(w) \\
& =\int_{\mathbb{D}} K_{\mathbb{D}}\left(w, \varphi^{-1}(z)\right) \frac{1}{\varphi^{\prime}(w)} \overline{\left(\varphi^{-1}\right)^{\prime}(z)} f \circ \varphi(w)\left|\varphi^{\prime}(w)\right|^{2} d A(w)
\end{aligned}
$$

(by change of variable)

$$
\begin{aligned}
& =\overline{\left(\varphi^{-1}\right)^{\prime}(z)} \int_{\mathbb{D}} K_{\mathbb{D}}\left(w, \varphi^{-1}(z)\right) \frac{\overline{\varphi^{\prime}(w)}}{\varphi^{\prime}(w)} f \circ \varphi(w) \varphi^{\prime}(w) d A(w) \\
& =\left(V_{\varphi^{-1}} h_{\overline{\varphi^{\prime}} / \varphi^{\prime}} U_{\varphi} f\right)(z) .
\end{aligned}
$$

Thus

$$
\bar{T}_{\Omega}=V_{\varphi^{-1}} h_{\overline{\varphi^{\prime}} / \varphi^{\prime}} U_{\varphi} .
$$

The second equality follows from the following property of the small Hankel operator:

$$
h_{b}=h_{\overline{P_{\mathbf{D}} b}} \quad \text { for } \quad b \in L^{\infty}(\mathbb{D}) .
$$

By using Lemma 2.1, we can get the following compactness result.

Theorem 2.2. Let $\Omega$ be a proper, simply connected domain in $\mathbb{C}^{1}$ and let $\varphi$ be the conformal mapping of $\mathbb{D}$ onto $\Omega$. Then $T_{\Omega}$ is compact if and only if $P_{\mathbb{D}}\left(\varphi^{\prime} / \overline{\varphi^{\prime}}\right) \in \mathscr{B}_{0}(\mathbb{D})$, where $\mathscr{B}_{0}(\mathbb{D})$ is the little Bloch space which consists of holomorphic functions $f$ on $\mathbb{D}$ such that $\left(1-|z|^{2}\right) f^{\prime}(z) \rightarrow 0$ as $|z| \rightarrow 1^{-}$.

Proof. Since $T_{\Omega}$ is compact if and only if $\bar{T}_{\Omega}$ is compact, we only need to consider $\bar{T}_{\Omega}$. 
Since $U_{\varphi}: L_{a}^{2}(\Omega) \rightarrow L_{a}^{2}(\mathbb{D})$ and $V_{\varphi^{-1}}: \overline{L_{a}^{2}(\mathbb{D})} \rightarrow \overline{L_{a}^{2}(\Omega)}$ are unitary operators, by Lemma 2.1 we have that $\bar{T}_{\Omega}$ is compact if and only if the small Hankel operator $h_{\overline{P_{\mathrm{D}}\left(\varphi^{\prime} / \overline{\varphi^{\prime}}\right)}}$ is compact. But by Corollary 2 of Theorem 7.6.6 in [Zh], we know that $h_{P_{\mathbb{D}}\left(\varphi^{\prime} / \overline{\varphi^{\prime}}\right)}$ is compact if and only if $P_{\mathbb{D}}\left(\varphi^{\prime} / \overline{\varphi^{\prime}}\right) \in \mathscr{B}_{0}(\mathbb{D})$. This completes the proof.

From Theorem 2.2 we have

Theorem 2.3. Let $\Omega$ be a proper, simply connected domain in $\mathbb{C}^{1}$ and let $\varphi$ be the conformal mapping of $\mathbb{D}$ onto $\Omega$. If $\arg \left(\varphi^{\prime}\right) \in C(\overline{\mathbb{D}})$, then $T_{\Omega}$ is compact. Proof. If $\arg \left(\varphi^{\prime}\right) \in C(\overline{\mathbb{D}})$, we then have $\varphi^{\prime} / \overline{\varphi^{\prime}}=\mathrm{e}^{2 \arg \left(\varphi^{\prime}\right)} \in C(\overline{\mathbb{D}})$. By Theorem 5.2.5 of $[\mathrm{Zh}], P_{\mathbb{D}}$ maps $C(\overline{\mathbb{D}})$ onto $\mathscr{B}_{0}(\mathbb{D})$. Thus $P_{\mathbb{D}}\left(\varphi^{\prime} / \overline{\varphi^{\prime}}\right) \in \mathscr{B}_{0}(\mathbb{D})$. Then by Theorem 2.2, $T_{\Omega}$ is compact.

As a corollary of Theorem 2.3, we can get the following Friedrichs' compactness theorem $[\mathrm{F}]$.

Corollary $2.4([\mathrm{~F}])$. If $\Omega$ is a bounded simply connected domain in $\mathbb{C}^{1}$ with $C^{1}$ boundary, then $T_{\Omega}$ is compact.

The proof of Corollary 2.4 follows immediately from Theorem 2.3 and the following lemma.

Lemma 2.5. Let $\varphi$ map $\mathbb{D}$ conformally onto the inner domain of the Jordan curve $\Gamma$. Then $\Gamma$ is of class $C^{1}$ if and only if $\arg \left(\varphi^{\prime}\right)$ has a continuous extension to $\overline{\mathbb{D}}$.

Proof. See Theorem 3.2 of [Po].

Given $b \in L^{\infty}(\mathbb{D})$, the big Hankel operator $H_{b}$ (with symbol $b$ ) is defined by $H_{b} f=\left(I-P_{\mathbb{D}}\right)(b f)$ for $f \in L_{a}^{2}(\mathbb{D})$. Since the small Hankel operator $h_{b}$ is compact whenever the big Hankel operator $H_{b}$ is, by using the compactness result of the big Hankel operator $H_{b}$, we can also obtain the following sufficient condition for the compactness of the Friedrichs operator $T_{\Omega}$.

Theorem 2.6. Let $\Omega$ be a proper, simply connected domain in $\mathbb{C}^{1}$ and let $\varphi$ be the conformal mapping of $\mathbb{D}$ onto $\Omega$. If $\log \varphi^{\prime} \in \mathscr{B}_{0}(\mathbb{D})$, then $T_{\Omega}$ is compact.

Proof. Equivalently, we only need to prove that $\bar{T}_{\Omega}$ is compact. By Lemma 2.1 , we know that $\bar{T}_{\Omega}$ is compact if and only if the small Hankel operator $h_{\overline{\varphi^{\prime}} / \varphi^{\prime}}$ is compact. Now we consider the big Hankel operator $H_{\overline{\varphi^{\prime}} / \varphi^{\prime}}$. Since the symbol function $\overline{\varphi^{\prime}} / \varphi^{\prime}$ is smooth, by Theorem 2 of $[\mathrm{Lu}]$ we have that $H_{\overline{\varphi^{\prime}} / \varphi^{\prime}}$ is compact if the following condition is satisfied:

$$
\left(1-|z|^{2}\right) \frac{\partial}{\partial \bar{z}}\left(\frac{\overline{\varphi^{\prime}}}{\varphi^{\prime}}\right)(z) \rightarrow 0 \quad \text { as } \quad|z| \rightarrow 1 .
$$

But

$$
\left|\frac{\partial}{\partial \bar{z}} \frac{\overline{\varphi^{\prime}}}{\varphi^{\prime}}\right|=\left|\frac{\overline{\varphi^{\prime \prime}} \varphi^{\prime}}{\left(\varphi^{\prime}\right)^{2}}\right|=\left|\frac{\varphi^{\prime \prime}}{\varphi^{\prime}}\right|=\left|\left(\log \varphi^{\prime}\right)^{\prime}\right| .
$$

Therefore condition (2.1) is equivalent to $\log \varphi^{\prime} \in \mathscr{B}_{0}(\mathbb{D})$. Thus, if $\log \varphi^{\prime} \in$ $\mathscr{B}_{0}(\mathbb{D})$, then the big Hankel operator $H_{\overline{\varphi^{\prime}} / \varphi^{\prime}}$ is compact, and this implies that the small Hankel operator $h_{\overline{\varphi^{\prime}} / \varphi^{\prime}}$ is compact. Hence $\bar{T}_{\Omega}$ is compact. This completes the proof. 


\section{SCHATTEN CLASS FRIEDRICHS OPERATORS}

First, we need to collect a couple of definitions.

For $1<p<\infty$, the holomorphic Besov space $B_{p}(\mathbb{D})$ on the unit disk is defined to be the space of holomorphic functions $f$ on $\mathbb{D}$ such that

$$
\|f\|_{B_{p}(\mathbb{D})}=\left(\int_{\mathbb{D}}\left(1-|z|^{2}\right)^{p-2}\left|f^{\prime}(z)\right|^{p} d A(z)\right)^{1 / p}<\infty .
$$

For $p=1, B_{1}(\mathbb{D})$ is the space of holomorphic functions $f$ on $\mathbb{D}$ which can be written as

$$
f(z)=\sum_{n=1}^{+\infty} a_{n} \frac{\lambda_{n}-z}{1-\bar{\lambda}_{n} z}
$$

for some sequence $\left\{a_{n}\right\}$ in $l^{1}$ and $\left\{\lambda_{n}\right\}$ in $\mathbb{D}$.

The weighted holomorphic Sobolev space $\mathscr{W}_{p}^{s, \alpha}(\mathbb{D})$ on the unit disk is the subspace of $W_{p}^{s, \alpha}(\mathbb{D})$ (defined in Section 1) consisting of holomorphic functions on $\mathbb{D}$.

Now we can consider the membership of $\bar{T}_{\Omega}$ in the Schatten classes $S_{p}$.

Theorem 3.1. Let $\Omega$ be a proper, simply connected domain in $\mathbb{C}^{1}$ and let $\varphi$ be the conformal mapping of $\mathbb{D}$ onto $\Omega$. Then for $1 \leq p<\infty, \bar{T}_{\Omega} \in S_{p}$ if and only if $P_{\mathbb{D}}\left(\varphi^{\prime} / \overline{\varphi^{\prime}}\right) \in B_{p}(\mathbb{D})$.

Proof. Since $U_{\varphi}: L_{a}^{2}(\Omega) \rightarrow L_{a}^{2}(\mathbb{D})$ and $V_{\varphi^{-1}}: \overline{L_{a}^{2}(\mathbb{D})} \rightarrow \overline{L_{a}^{2}(\Omega)}$ are unitary

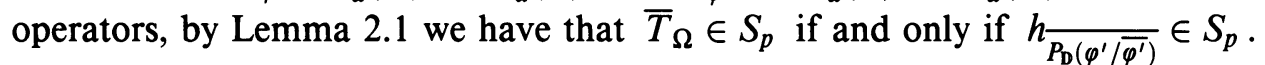

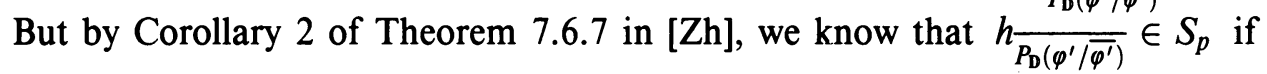
and only if $P_{\mathbb{D}}\left(\varphi^{\prime} / \overline{\varphi^{\prime}}\right) \in B_{p}(\mathbb{D})$. This completes the proof.

In order to get our main theorem, we need the following lemma.

Lemma 3.2. Let $s \in \mathbb{Z}_{+}$and $1 \leq p<\infty$. Then for any $0<\gamma<p, P_{\mathbb{D}}$ projects $W_{p}^{s, \gamma}(\mathbb{D})$ continuously onto its holomorphic subspace $\mathscr{W}_{p}^{s, \gamma}(\mathbb{D})$.

Proof. See [BB]. This lemma is a special case of Corollary 6.3(ii) in [BB].

Now we are ready for our main theorem.

Main Theorem. Let $\Omega$ be a proper, simply connected domain in $\mathbb{C}^{1}$ and let $\varphi$ be the conformal mapping of $\mathbb{D}$ onto $\Omega$. Then:

(1) For $1<p<\infty$, if $\varphi^{\prime} / \overline{\varphi^{\prime}} \in W_{p}^{1, p-1}(\mathbb{D})$, then $\bar{T}_{\Omega} \in S_{p}$.

(2) For $p=1$, if $\varphi^{\prime} / \overline{\varphi^{\prime}} \in W_{1}^{2, \gamma}(\mathbb{D})$ for some $0<\gamma<1$, then $\bar{T}_{\Omega} \in S_{1}$.

Proof. First we prove (1). For $1<p<\infty$, using Lemma 3.2, we have

$$
\left\|P_{\mathbb{D}} f\right\|_{\mathscr{W}_{p}^{1, p-1}(\mathbb{D})} \leq C\|f\|_{W_{p}^{1, p-1}(\mathbb{D})} \quad \text { for } \quad f \in W_{p}^{1, p-1}(\mathbb{D}) .
$$

Then if $\varphi^{\prime} / \overline{\varphi^{\prime}} \in W_{p}^{1, p-1}(\mathbb{D})$, we have

$$
\begin{aligned}
\left\|P_{\mathbb{D}}\left(\varphi^{\prime} / \overline{\varphi^{\prime}}\right)\right\|_{B_{p}(\mathbb{D})} & =\left(\int_{\mathbb{D}}\left|\frac{\partial}{\partial z}\left(P_{\mathbb{D}}\left(\varphi^{\prime} / \overline{\varphi^{\prime}}\right)\right)\right|^{p}\left(1-|z|^{2}\right)^{p-2} d A\right)^{1 / p} \\
& \leq\left\|P_{\mathbb{D}}\left(\varphi^{\prime} / \overline{\varphi^{\prime}}\right)\right\|_{\mathscr{W}_{p}^{1, p-1}(\mathbb{D})} \\
& \leq C\left\|\varphi^{\prime} / \overline{\varphi^{\prime}}\right\|_{W_{p}^{1, p-1}(\mathbb{D})}<\infty .
\end{aligned}
$$


Thus $P_{\mathbb{D}}\left(\varphi^{\prime} / \overline{\varphi^{\prime}}\right) \in B_{p}(\mathbb{D})$. It then follows from Theorem 3.1 that $\bar{T}_{\Omega} \in S_{p}$.

Now we prove (2). For $p=1$, using Lemma 3.2, we have for any $0<\gamma<1$,

$$
\left\|P_{\mathbb{D}} f\right\|_{\mathscr{W}_{1}^{2, \gamma(\mathbb{D})}} \leq C\|f\|_{W_{1}^{2, \gamma}(\mathbb{D})} \quad \text { for } \quad f \in W_{1}^{2, \gamma}(\mathbb{D}) .
$$

On the other hand, it is well known that $g \in B_{1}(\mathbb{D})$ if and only if

$$
\int_{\mathbb{D}}\left|g^{\prime \prime}(z)\right| d A(z)<\infty .
$$

Then if $\varphi^{\prime} / \overline{\varphi^{\prime}} \in W_{1}^{2, \gamma}(\mathbb{D})$ for some $0<\gamma<1$, we have

$$
\begin{aligned}
\int_{\mathbb{D}}\left|\frac{\partial^{2}}{\partial z^{2}}\left(P_{\mathbb{D}}\left(\varphi^{\prime} / \overline{\varphi^{\prime}}\right)\right)\right| d A(z) & \leq \int_{\mathbb{D}}\left|\frac{\partial^{2}}{\partial z^{2}}\left(P_{\mathbb{D}}\left(\varphi^{\prime} / \overline{\varphi^{\prime}}\right)\right)\right|\left(1-|z|^{2}\right)^{\gamma-1} d A(z) \\
& \leq\left\|P_{\mathbb{D}}\left(\varphi^{\prime} / \overline{\varphi^{\prime}}\right)\right\|_{\mathscr{W}_{1}^{2, \gamma}(\mathbb{D})} \\
& \leq C\left\|\varphi^{\prime} / \overline{\varphi^{\prime}}\right\|_{W_{1}^{2, \gamma}(\mathbb{D})}<\infty .
\end{aligned}
$$

Thus $P_{\mathbb{D}}\left(\varphi^{\prime} / \overline{\varphi^{\prime}}\right) \in B_{1}(\mathbb{D})$. It then follows from Theorem 3.1 that $\bar{T}_{\Omega} \in S_{1}$. This completes the proof of (2) and of the theorem.

From the Main Theorem we have several corollaries.

Corollary 3.3. Let $\Omega$ be a proper, simply connected domain in $\mathbb{C}^{1}$ and let $\varphi$ be the conformal mapping of $\mathbb{D}$ onto $\Omega$. Then for $1<p<\infty$, if $\log \varphi^{\prime} \in B_{p}(\mathbb{D})$, then $\bar{T}_{\Omega} \in S_{p}$.

Proof. If $\log \varphi^{\prime} \in B_{p}(\mathbb{D})$ with $1<p<\infty$, then

$$
\begin{aligned}
\left\|\varphi^{\prime} / \overline{\varphi^{\prime}}\right\|_{W_{p}^{1, p-1}(\mathbb{D})}^{p}= & \int_{\mathbb{D}}\left(1-|z|^{2}\right)^{p-2}\left|\varphi^{\prime} / \overline{\varphi^{\prime}}\right|^{p} d A(z) \\
& +\int_{\mathbb{D}}\left(1-|z|^{2}\right)^{p-2}\left|\frac{\partial}{\partial z}\left(\varphi^{\prime} / \overline{\varphi^{\prime}}\right)\right|^{p} d A(z) \\
& +\int_{\mathbb{D}}\left(1-|z|^{2}\right)^{p-2}\left|\frac{\partial}{\partial \bar{z}}\left(\varphi^{\prime} / \overline{\varphi^{\prime}}\right)\right|^{p} d A(z) \\
= & C+\int_{\mathbb{D}}\left(1-|z|^{2}\right)^{p-2}\left|\frac{\varphi^{\prime \prime} \overline{\varphi^{\prime}}}{{\overline{\varphi^{\prime}}}^{2}}\right|^{p} d A(z) \\
& +\int_{\mathbb{D}}\left(1-|z|^{2}\right)^{p-2}\left|\frac{-{\overline{\varphi^{\prime \prime}}}^{\prime}}{{\overline{\varphi^{\prime}}}^{2}}\right|^{p} d A(z) \\
= & C+2 \int_{\mathbb{D}}\left(1-|z|^{2}\right)^{p-2}\left|\frac{\varphi^{\prime \prime}}{\varphi^{\prime}}\right|^{p} d A(z) \\
= & C+2\left\|\log \varphi^{\prime}\right\|_{B_{p}(\mathbb{D})}^{p}<\infty .
\end{aligned}
$$

Thus $\varphi^{\prime} / \overline{\varphi^{\prime}} \in W_{p}^{1, p-1}(\mathbb{D})$. It then follows from (1) of the Main Theorem that $\bar{T}_{\Omega} \in S_{p}$.

Corollary A. If $\Omega$ is a bounded simply connected domain in $\mathbb{C}^{1}$ with $C^{2, \alpha}(0<$ $\alpha<1)$ boundary, then $\bar{T}_{\Omega}$ belongs to all $S_{p}$ for $1<p<\infty$.

To prove Corollary A, we need the following lemma. 
Lemma 3.4. Let $\varphi$ map $\mathbb{D}$ conformally onto the inner domain of the Jordan curve $\Gamma$ of class $C^{n, \alpha}$ where $n=1,2, \cdots$ and $0<\alpha<1$. Then $\varphi^{(n)}$ has $a$ continuous extension to $\overline{\mathbb{D}}$ and $\varphi^{\prime}(z) \neq 0$ for $z \in \overline{\mathbb{D}}$.

Proof. See Theorems 3.5 and 3.6 of [Po].

Proof of Corollary A. Let $\varphi$ be the conformal mapping of $\mathbb{D}$ onto $\Omega$. By Lemma 3.4, we have that $\varphi \in C^{2}(\overline{\mathbb{D}})$ and $\varphi^{\prime}(z) \neq 0$ for $z \in \overline{\mathbb{D}}$. Hence $\varphi^{\prime} / \overline{\varphi^{\prime}} \in C^{1}(\overline{\mathbb{D}})$. It is easy to check that $C^{1}(\overline{\mathbb{D}}) \subset W_{p}^{1, p-1}(\mathbb{D})(1<p<\infty)$. It then follows from (1) of the Main Theorem that $\bar{T}_{\Omega} \in S_{p}$ for all $1<p<\infty$.

Corollary B. If $\Omega$ is a bounded simply connected domain in $\mathbb{C}^{1}$ with $C^{3, \alpha}(0<$ $\alpha<1)$ boundary, then $\bar{T}_{\Omega} \in S_{1}$.

Proof. Let $\varphi$ be the conformal mapping of $\mathbb{D}$ onto $\Omega$. By Lemma 3.4, we have that $\varphi \in C^{3}(\overline{\mathbb{D}})$ and $\varphi^{\prime}(z) \neq 0$ for $z \in \overline{\mathbb{D}}$. Hence $\varphi^{\prime} / \overline{\varphi^{\prime}} \in C^{2}(\overline{\mathbb{D}})$. It is easy to see that $C^{2}(\overline{\mathbb{D}}) \subset W_{1}^{2, \gamma}(\mathbb{D})$ for any $0<\gamma<1$. It then follows from (2) of the Main Theorem that $\bar{T}_{\Omega} \in S_{1}$.

\section{4. АвоUт $T_{\Omega}^{2}$}

As we pointed out in the introduction, $T_{\Omega}$ is real-linear but not complexlinear. Sometimes (as in [F] and [N]) it is convenient to study $T_{\Omega}^{2}$ instead of $T_{\Omega} . T_{\Omega}^{2}$ is a complex-linear positive (hence in particular selfadjoint) operator on $L_{a}^{2}(\Omega)$. When $T_{\Omega}$ is compact, $T_{\Omega}^{2}$ has a sequence of eigenvalues $1=\lambda_{0}>\lambda_{1} \geq \lambda_{2} \ldots$ decreasing to zero, and a corresponding sequence of eigenfunctions $\left\{\varphi_{n}(z)\right\}_{n \geq 0}$ which form an orthonormal basis for $L_{a}^{2}(\Omega)$ and satisfy the remarkable "double orthogonality" relations

$$
\int_{\Omega} \varphi_{m} \bar{\varphi}_{n} d A=0=\int_{\Omega} \varphi_{m} \varphi_{n} d A \quad(m \neq n) .
$$

See [Sh2] for a more detailed discussion about $T_{\Omega}^{2}$.

Observe that for $f \in L_{a}^{2}(\Omega), T_{\Omega}^{2} f=P_{\Omega}\left(\overline{T_{\Omega} f}\right)=P_{\Omega}\left(\bar{T}_{\Omega} \hat{J}\right)$. Hence $T_{\Omega}^{2}=$ $P_{\Omega} \bar{T}_{\Omega}$. Thus $T_{\Omega}^{2} \in S_{p}$ whenever $\bar{T}_{\Omega} \in S_{p}$. Then from our main theorem we have

Theorem 4.1. Let $\Omega$ be a proper, simply connected domain in $\mathbb{C}^{1}$ and let $\varphi$ be the conformal mapping of $\mathbb{D}$ onto $\Omega$. Then:

(1) For $1<p<\infty$, if $\varphi^{\prime} / \overline{\varphi^{\prime}} \in W_{p}^{1, p-1}(\mathbb{D})$, then $T_{\Omega}^{2} \in S_{p}$.

(2) For $p=1$, if $\varphi^{\prime} / \overline{\varphi^{\prime}} \in W_{1}^{2, \gamma}(\mathbb{D})$ for some $0<\gamma<1$, then $T_{\Omega}^{2} \in S_{1}$.

Similarly, Corollaries 3.3, A and B are also valid for $T_{\Omega}^{2}$.

\section{ACKNOWLEDGMENT}

We would like to thank the referee for several helpful suggestions.

\section{REFERENCES}

[B] S. Bergman, The kernel function and conformal mapping, Math. Surveys Monographs, vol. 5, Amer. Math. Soc., Providence, RI, 1950.

[BB] F. Beatrous and J. Burbea, Holomorphic Sobolev spaces on the ball, Dissertationes Math. (Rozprawy Mat.) 276 (1989). 
[F] K. Friedrichs, On certain inequalities for analytic functions and for functions of two variables, Trans. Amer. Math. Soc. 41 (1937), 321-364.

[Lu] D. Luecking, Characterizations of certain classes of Hankel operators on the Bergman spaces of the unit disk, J. Funct. Anal. 110 (1992), 247-271.

[N] S. Norman, The Friedrichs operator, research report, TRITAMAT, Royal Inst. of Technology 6 (1987).

[Po] Ch. Pommerenke, Boundary behavious of conformal maps, Springer-Verlag, New York, 1992.

[Sh] H. S. Shapiro, Domains allowing exact quadrature identities for harmonic functions-an approach based on p.d.e., Anniversary Volume on Approximation Theory and Functional Analysis, Internat. Ser. Numer. Math., vol. 65, Birkhäuser Verlag, Basel, 1984, pp. 335-354.

[Sh2] _ The Schwarz function and its generalization to higher dimensions, Wiley, New York, 1993.

[Zh] Kehe Zhu, Operator theory in function spaces, Marcel Dekker, New York, 1990.

Department of Mathematics, Washington University, St. Louis, Missouri 63130

E-mail address: plin@math. wustl.edu

E-mail address: rr@jezebel. wustl.edu 